\title{
POSITIVE EXPRESSIONS FOR SKEW DIVIDED DIFFERENCE OPERATORS
}

\author{
RICKY INI LIU
}

\begin{abstract}
For permutations $v, w \in \mathfrak{S}_{n}$, Macdonald defines the skew divided difference operators $\partial_{w / v}$ as the unique linear operators satisfying $\partial_{w}(P Q)=\sum_{v} v\left(\partial_{w / v} P\right) \cdot \partial_{v} Q$ for all polynomials $P$ and $Q$. We prove that $\partial_{w / v}$ has a positive expression in terms of divided difference operators $\partial_{i j}$ for $i<j$. In fact, we prove that the analogous result holds in the Fomin-Kirillov algebra $\mathcal{E}_{n}$, which settles a conjecture of Kirillov.
\end{abstract}

\section{INTRODUCTION}

The divided difference operators $\partial_{i j}$ acting on $\mathbf{C}\left[x_{1}, \ldots, x_{n}\right]$ are vital in the study of Schubert calculus. Their main purpose is to define Schubert polynomials, which serve as polynomial representatives of Schubert classes in the cohomology ring of the flag variety. Finding a combinatorial formula for the structure constants $c_{u v}^{w}$ of Schubert polynomials is a long outstanding problem in algebraic combinatorics.

Macdonald [11] defined for any permutations $v$ and $w$ a skew divided difference operator $\partial_{w / v}$ such that applying $\partial_{w / v}$ to the Schubert polynomial of a permutation $u$ with $\ell(u)+\ell(v)=$ $\ell(w)$ gives the structure constant $c_{u v}^{w}$. In [9], Kirillov conjectures that $\partial_{w / v}$ can be written as a polynomial in $\partial_{i j}$ for $i<j$ with positive coefficients. The main result of this paper is to prove this conjecture.

In fact, Kirillov conjectures a slightly more general result. The divided difference operators give a representation of a larger algebra $\mathcal{E}_{n}$ introduced by Fomin and Kirillov [5]. Kirillov then conjectures that the element of the Fomin-Kirillov algebra corresponding to $\partial_{w / v}$ has a positive expression in terms of generators $x_{i j} \in \mathcal{E}_{n}$ for $i<j$. This form of positivity in $\mathcal{E}_{n}$ is notable due to the nonnegativity conjecture in [5], which states that certain elements of $\mathcal{E}_{n}$ (namely evaluations of Schubert polynomials at Dunkl elements) have such a positive expression. A proof of this nonnegativity conjecture together with an explicit positive expression for such elements would immediately give a combinatorial formula for the structure constants $c_{u v}^{w}$.

The Fomin-Kirillov algebra also has the structure of a braided Hopf algebra as noted in [6, 12. This added structure (which does not exist in full for the quotient algebra generated by the divided difference operators) will be key in proving our main theorem.

We begin with some preliminaries about the symmetric group, divided difference operators, and the braided Hopf algebra structure of the Fomin-Kirillov algebra $\mathcal{E}_{n}$ in Section 2. We then prove the main result in Theorem [3.5 of Section 3, giving a positive explicit formula for $\partial_{w / v}$ in Corollary 3.6 and a positive recursive formula in Corollary 3.8, 


\section{Preliminaries}

In this section, we give some notation and background, and we prove some basic facts about divided difference operators and the Fomin-Kirillov algebra. For more information, see, for instance, [8, 9, 11].

2.1. Symmetric group. Let $\mathfrak{S}_{n}$ be the symmetric group on $n$ letters. We will write $s_{i j}$ for the transposition switching $i$ and $j$, and we will abbreviate the simple transposition $s_{i, i+1}$ by $s_{i}$.

Given an element $w \in \mathfrak{S}_{n}$, a reduced expression for $w$ is a factorization of $w$ into simple transpositions $s_{i_{1}} \cdots s_{i_{\ell}}$ of minimum length $\ell=\ell(w)$. Any two reduced expressions for $w$ can be obtained from one another by commuting $s_{i}$ and $s_{j}$ when $|i-j|>1$ or by applying braid moves replacing $s_{i} s_{j} s_{i}$ with $s_{j} s_{i} s_{j}$ when $|i-j|=1$.

We say $w=u \cdot v$ is a reduced factorization if $\ell(w)=\ell(u)+\ell(v)$. We also denote the longest element of $\mathfrak{S}_{n}$ by $w_{0}$, so $\ell\left(w_{0}\right)=\left(\begin{array}{l}n \\ 2\end{array}\right)$.

If some (equivalently, any) reduced expression for $w$ contains a subsequence that is a reduced expression for $v$, we say that $v<w$ in Bruhat order. Equivalently, $v$ is covered by $w$ in Bruhat order, written $v \lessdot w$, if $\ell(v)=\ell(w)-1$ and $v=w s_{i j}$ for some transposition $s_{i j}$.

2.2. Divided difference operators. Define the left action of $\mathfrak{S}_{n}$ on $\mathbf{C}\left[x_{1}, \ldots, x_{n}\right]$ by

$$
(w P)\left(x_{1}, \ldots, x_{n}\right)=P\left(x_{w(1)}, \ldots, x_{w(n)}\right) .
$$

The divided difference operator $\partial_{i j}$ is then defined by

$$
\partial_{i j} P=\frac{P-s_{i j} P}{x_{i}-x_{j}}
$$

for distinct $i$ and $j$. We abbreviate $\partial_{i, i+1}$ by $\partial_{i}$.

The following proposition describes some simple but important properties of the divided difference operators.

Proposition 2.1. The divided difference operators satisfy, for distinct $i, j, k$ and $l$ :

(a) $\partial_{i j}=-\partial_{j i}$;

(b) $\partial_{i j}^{2}=0$;

(c) $\partial_{i j} \partial_{k l}=\partial_{k l} \partial_{i j}$;

(d) $\partial_{i j} \partial_{j k}+\partial_{j k} \partial_{k i}+\partial_{k i} \partial_{i j}=0$;

(e) $\partial_{i j} \partial_{j k} \partial_{i j}=\partial_{j k} \partial_{i j} \partial_{j k}=\partial_{i j} \partial_{i k} \partial_{j k}=\partial_{j k} \partial_{i k} \partial_{i j}$;

(f) $\partial_{i j}(P Q)=\partial_{i j} P \cdot Q+s_{i j} P \cdot \partial_{i j} Q$; and

(g) $\partial_{i j} w=w \partial_{w^{-1}(i) w^{-1}(j)}$ for all $w \in \mathfrak{S}_{n}$.

Proof. Straightforward computation.

Proposition 2.1(e) follows from (a), (b), and (d). The first equality is the braid relation, while the last equality is sometimes called the Yang-Baxter equation.

The divided difference operators satisfy other relations that are not implied by those in Proposition 2.1, but we will not need them here. However, the only relations between the simple divided difference operators $\partial_{i}$ are (b), (c), and the braid relation in (e). These relations define the nil-Coxeter algebra of $\mathfrak{S}_{n}$. Thus $\partial_{1}, \ldots, \partial_{n-1}$ generate a faithful representation of the nil-Coxeter algebra (see [7]). Given a permutation $w \in \mathfrak{S}_{n}$, let $w=s_{i_{1}} \cdots s_{i_{\ell}}$ be a reduced expression, and define $\partial_{w}=\partial_{i_{1}} \cdots \partial_{i_{\ell}}$. Then $\partial_{w}$ does not depend on the choice of reduced 
expression for $w$. For any two permutations $v, w \in \mathfrak{S}_{n}, \partial_{v} \partial_{w}=\partial_{v w}$ if $\ell(v w)=\ell(v)+\ell(w)$ and 0 otherwise.

By applying Proposition 2.1(f) repeatedly to $\partial_{w}(P Q)=\partial_{i_{1}} \cdots \partial_{i_{\ell}}(P Q)$, we can express $\partial_{w}(P Q)$ as

$$
\partial_{w}(P Q)=\sum_{v \in \mathfrak{S}_{n}} v\left(\partial_{w / v} P\right) \cdot \partial_{v} Q
$$

for some linear operators $\partial_{w / v}$. These operators are called the skew divided difference operators. The operator $\partial_{w / v}$ reduces the degree of a polynomial by $\ell(w)-\ell(v)$.

Example 2.2. Let $w=s_{1} s_{2} \in \mathfrak{S}_{3}$. Then

$$
\begin{aligned}
\partial_{w}(P Q) & =\partial_{1} \partial_{2}(P Q) \\
& =\partial_{1}\left(\partial_{2} P \cdot Q+s_{2} P \cdot \partial_{2} Q\right) \\
& =\partial_{1} \partial_{2} P \cdot Q+s_{1} \partial_{2} P \cdot \partial_{1} Q+\partial_{1} s_{2} P \cdot \partial_{2} Q+s_{1} s_{2} P \cdot \partial_{1} \partial_{2} Q \\
& =\partial_{1} \partial_{2} P \cdot Q+s_{1} \partial_{2} P \cdot \partial_{1} Q+s_{2} \partial_{13} P \cdot \partial_{2} Q+s_{1} s_{2} P \cdot \partial_{1} \partial_{2} Q .
\end{aligned}
$$

Hence $\partial_{w / i d}=\partial_{1} \partial_{2}, \partial_{w / s_{1}}=\partial_{2}, \partial_{w / s_{2}}=\partial_{13}$, and $\partial_{w / w}=1$.

Alternatively, $\partial_{w / v}$ can be calculated as follows. Again consider any reduced expression $w=s_{i_{1}} \ldots s_{i_{\ell}}$. For any subset $J \subseteq\{1, \ldots, \ell\}$, let $\varphi_{J}=\prod_{j=1}^{\ell} \varphi_{j}(J)$, where $\varphi_{j}(J)=s_{i_{j}}$ if $j \in J$ and $\partial_{i_{j}}$ if $j \notin J$. Then

$$
\partial_{w / v}=v^{-1} \sum_{J} \varphi_{J}
$$

where $J$ ranges over all subsets for which the product $\prod_{j \in J} s_{i_{j}}$ is a reduced expression for $v$. Note that this immediately implies that $\partial_{w / v}=0$ unless $v<w$ in Bruhat order.

By using Proposition 2.1(g) to collect the transpositions in $\varphi_{J}$ to the left, we will always be able to write $\partial_{w / v}$ as a polynomial in the divided difference operators $\partial_{i j}$.

Example 2.3. Let $w=s_{2} s_{1} s_{3} s_{2} \in \mathfrak{S}_{4}$, and let $v=s_{2}$. Then the only possibilities for $J$ that yield a reduced expression for $v$ are $J=\{1\}$ and $J=\{4\}$. Thus

$$
\begin{aligned}
\partial_{w / v} & =v^{-1}\left(s_{2} \partial_{12} \partial_{34} \partial_{23}+\partial_{23} \partial_{12} \partial_{34} s_{2}\right) \\
& =s_{2}\left(s_{2} \partial_{12} \partial_{34} \partial_{23}+s_{2} \partial_{32} \partial_{13} \partial_{24}\right) \\
& =\partial_{12} \partial_{34} \partial_{23}-\partial_{23} \partial_{13} \partial_{24} .
\end{aligned}
$$

Note that while this expression has a negative sign in it, we can use Proposition 2.1(d) to rewrite it as

$$
\begin{aligned}
\partial_{12} \partial_{34} \partial_{23}-\partial_{23} \partial_{13} \partial_{24} & =\partial_{12}\left(\partial_{23} \partial_{24}+\partial_{24} \partial_{34}\right)-\left(\partial_{12} \partial_{23}-\partial_{13} \partial_{12}\right) \partial_{24} \\
& =\partial_{12} \partial_{24} \partial_{34}+\partial_{13} \partial_{12} \partial_{24}
\end{aligned}
$$

This example shows that naïve evaluation of $\partial_{w / v}$ will generally not give an expression in $\partial_{i j}$ with $i<j$ that has positive coefficients. The main result of this paper will be to prove the following theorem, which states that such a positive expression always exists. It will follow as an immediate consequence of Theorem 3.5 below.

Theorem 2.4. For any $v, w \in \mathfrak{S}_{n}$, the skew divided difference operator $\partial_{w / v}$ can be written as a polynomial with nonnegative coefficients in the operators $\partial_{i j}$ for $i<j$.

An explicit expression for $\partial_{w / v}$ follows from Corollary 3.6 . 
2.3. Fomin-Kirillov algebra. We will not need the full set of relations between the $\partial_{i j}$ to prove Theorem 2.4, only the ones appearing in Proposition 2.1. As such, it will be helpful to work inside the Fomin-Kirillov algebra $\mathcal{E}_{n}$, which is essentially defined by the relations in Proposition [2.1(a)-(d). (Relation (e) can be derived from these four.) See [3, 5] for more information.

Definition. The Fomin-Kirillov algebra $\mathcal{E}_{n}$ is the (noncommutative) algebra with generators $x_{i j}=-x_{j i}$ for $1 \leq i<j \leq n$ satisfying the following relations for distinct $i, j, k$, and $l$ :

- $x_{i j}^{2}=0$;

- $x_{i j} x_{k l}=x_{k l} x_{i j}$; and

- $x_{i j} x_{j k}+x_{j k} x_{k i}+x_{k i} x_{i j}=0$.

The divided difference operators therefore give a representation of $\mathcal{E}_{n}$, though this representation is not faithful for $n \geq 3$. As with divided difference operators, the subalgebra generated by $x_{12}, x_{23}, \ldots, x_{n-1, n}$ is isomorphic to the nil-Coxeter algebra. Given any $w \in \mathfrak{S}_{n}$ with reduced expression $w=s_{i_{1}} \cdots s_{i_{\ell}}$, we will define $x_{w}=x_{i_{1}, i_{1}+1} \cdots x_{i_{\ell}, i_{\ell}+1} \in \mathcal{E}_{n}$. We can similarly define $x_{w / v} \in \mathcal{E}_{n}$ as before (or one can take Proposition 2.7 below as a definition).

One important property of $\mathcal{E}_{n}$ is that it has a large amount of structure: in particular, it is a braided Hopf algebra. (This is not the case for the algebra generated by divided difference operators, which is a quotient of $\mathcal{E}_{n}$.) We review some properties of this structure below. For more details about this structure and braided Hopf algebras in general, see [1, 3, 6, 12,

2.3.1. Grading and braiding. In addition to the usual degree grading, the Fomin-Kirillov algebra has an $\mathfrak{S}_{n}$-grading: define the $\mathfrak{S}_{n}$-degree of $x_{i j}$ to be $s_{i j}$ and extend by multiplicativity. We will write $s_{P}$ for the $\mathfrak{S}_{n}$-degree of an $\mathfrak{S}_{n}$-homogeneous element $P \in \mathcal{E}_{n}$. We will use the word "homogeneous" to mean homogeneous with respect to both the usual grading and the $\mathfrak{S}_{n}$-grading.

The Fomin-Kirillov algebra also has an $\mathfrak{S}_{n}$-action given by permuting the indices of the generators $x_{i j}$. In other words, for $w \in \mathfrak{S}_{n}, w\left(x_{i j}\right)=x_{w(i) w(j)}$, and we extend by multiplicativity. This induces an automorphism of $\mathcal{E}_{n}$.

Using this gradation and action, $\mathcal{E}_{n}$ can be thought of as an object in a braided monoidal category (or more specifically, in the Yetter-Drinfeld category over $\mathbf{C}\left[S_{n}\right]$ ). In other words, we define a braiding $\tau: \mathcal{E}_{n} \otimes \mathcal{E}_{n} \rightarrow \mathcal{E}_{n} \otimes \mathcal{E}_{n}$ by $\tau(P \otimes Q)=Q \otimes s_{Q}^{-1}(P)$ for homogeneous $P, Q \in \mathcal{E}_{n}$. We use this to define a braided product structure on $\mathcal{E}_{n} \otimes \mathcal{E}_{n}$ via

$$
\left(P_{1} \otimes P_{2}\right)\left(Q_{1} \otimes Q_{2}\right)=P_{1} Q_{1} \otimes s_{Q_{1}}^{-1}\left(P_{2}\right) Q_{2} \text {. }
$$

Remark 2.5. This convention for the braiding is different from the usual convention, but we use it here for ease of compatibility with the definitions of skew divided difference operators.

2.3.2. Coproduct. There exists a coproduct $\Delta$ on $\mathcal{E}_{n}$ defined by $x_{i j} \mapsto x_{i j} \otimes 1+1 \otimes x_{i j}$ and extended to all of $\mathcal{E}_{n}$ as a braided homomorphism.

Example 2.6. We can compute $\Delta\left(x_{12} x_{23}\right)$ :

$$
\begin{aligned}
\Delta\left(x_{12} x_{23}\right) & =\left(x_{12} \otimes 1+1 \otimes x_{12}\right)\left(x_{23} \otimes 1+1 \otimes x_{23}\right) \\
& =\left(x_{12} \otimes 1\right)\left(x_{23} \otimes 1\right)+\left(x_{12} \otimes 1\right)\left(1 \otimes x_{23}\right)+\left(1 \otimes x_{12}\right)\left(x_{23} \otimes 1\right)+\left(1 \otimes x_{12}\right)\left(1 \otimes x_{23}\right) \\
& =x_{12} x_{23} \otimes 1+x_{12} \otimes x_{23}+x_{23} \otimes x_{13}+1 \otimes x_{12} x_{23} .
\end{aligned}
$$

Compare this calculation to Example 2.2. 
The main reason for introducing the coproduct structure is the following proposition.

Proposition 2.7. For any $w \in \mathfrak{S}_{n}, \Delta\left(x_{w}\right)=\sum_{v \in \mathfrak{S}_{n}} x_{v} \otimes x_{w / v}$.

Proof. Follows from the definition of $x_{w / v}$ and the coproduct structure.

Note that if $P \in \mathcal{E}_{n}$ is a monomial, then any term appearing in the first tensor factor of $\Delta(P)$ is the product of a subsequence of variables in $P$. (This is not the case for the second tensor factor due to the braiding.)

2.3.3. Pairing. There exists a unique linear map $\Delta_{a b}: \mathcal{E}_{n} \rightarrow \mathcal{E}_{n}$ satisfying

$$
\Delta_{a b}\left(x_{i j}\right)= \begin{cases}1, & \text { if } i=a, j=b \\ -1, & \text { if } i=b, j=a \\ 0, & \text { otherwise; }\end{cases}
$$

and $\Delta_{a b}(P Q)=\Delta_{a b}(P) \cdot Q+s_{a b}(P) \cdot \Delta_{a b}(Q)$ for all $P, Q \in \mathcal{E}_{n}$. The operators $\Delta_{a b}$ satisfy the relations of $\mathcal{E}_{n}$, so they define a left action of $\mathcal{E}_{n}$ on itself. We can think of $\Delta_{a b}$ as having degree -1 and $\mathfrak{S}_{n}$-degree $s_{a b}$. For any $P \in \mathcal{E}_{n}$, we will write $\Delta_{P}$ for the corresponding operator; in other words, if $P=x_{i_{1} j_{1}} \cdots x_{i_{k} j_{k}}$, then $\Delta_{P}=\Delta_{i_{1} j_{1}} \cdots \Delta_{i_{k} j_{k}}$, and we extend by linearity.

There is likewise a dual action: one can define $\nabla_{a b}: \mathcal{E}_{n} \rightarrow \mathcal{E}_{n}$ (acting on the right) satisfying $\left(x_{i j}\right) \nabla_{a b}=\Delta_{a b}\left(x_{i j}\right)$ and $(P Q) \nabla_{a b}=P \cdot(Q) \nabla_{a b}+(P)\left(s_{Q} \nabla_{a b}\right) \cdot Q$, where $s_{Q} \nabla_{a b}=\nabla_{s_{Q}(a) s_{Q}(b)}$ for homogeneous $P, Q \in \mathcal{E}_{n}$. Then the operators $\nabla_{a b}$ define a right action of $\mathcal{E}_{n}$ on itself. We define $\nabla_{P}$ for any $P \in \mathcal{E}_{n}$ as above.

Note that when $\nabla_{a b}$ acts on a monomial $P$, it results in a linear combination of monomials obtained from $P$ by removing a variable. In other words, $(P) \nabla_{a b}$, and in general $(P) \nabla_{Q}$, has an expression that only contains variables appearing in $P$. (This is not the case for the action of $\Delta_{a b}$ due to the twisting action.)

Example 2.8. Here are two computations involving these operators:

$$
\begin{aligned}
\Delta_{23}\left(x_{12} x_{23} x_{12}\right) & =\Delta_{23}\left(x_{12}\right) \cdot x_{23} x_{12}+x_{13} \cdot \Delta_{23}\left(x_{23}\right) \cdot x_{12}+x_{13} x_{32} \cdot \Delta_{23}\left(x_{12}\right) \\
& =x_{13} x_{12}, \\
\left(x_{12} x_{23} x_{12}\right) \nabla_{23} & =x_{12} x_{23} \cdot\left(x_{12}\right) \nabla_{23}+x_{12} \cdot\left(x_{23}\right) \nabla_{13} \cdot x_{12}+\left(x_{12}\right) \nabla_{12} \cdot x_{23} x_{12} \\
& =x_{23} x_{12} .
\end{aligned}
$$

If $P$ and $Q$ are homogeneous of the same degree, then $\Delta_{P}(Q)=\Delta_{Q}(P)=(P) \nabla_{Q}=$ $(Q) \nabla_{P}$. If we write $\langle P, Q\rangle=\Delta_{P}(Q)$, then this defines a symmetric bilinear form $\langle\cdot, \cdot\rangle$ on $\mathcal{E}_{n}$. With respect to this form, $\Delta_{P}$ and $\nabla_{P}$ are adjoint to right and left multiplication by $P$, respectively. If $P$ and $Q$ are homogeneous, then $\langle P, Q\rangle=0$ unless $\operatorname{deg} P=\operatorname{deg} Q$ and $s_{P}=s_{Q}^{-1}$.

An alternative way to describe $\Delta_{P}$ and $\nabla_{P}$ is in terms of this bilinear form and the coproduct. If $\Delta(Q)=\sum Q_{(1)}^{i} \otimes Q_{(2)}^{i}$, then

$$
\begin{aligned}
& \Delta_{P}(Q)=\sum\left\langle P, Q_{(1)}^{i}\right\rangle \cdot Q_{(2)}^{i}, \\
& (Q) \nabla_{P}=\sum Q_{5}^{i} \cdot\left\langle Q_{(2)}^{i}, P\right\rangle .
\end{aligned}
$$


Note that by the cocommutativity of the coproduct, $\Delta_{P_{1}}$ and $\nabla_{P_{2}}$ commute: if $(\Delta \otimes \Delta)(Q)=$ $\sum Q_{(1)}^{i} \otimes Q_{(2)}^{i} \otimes Q_{(3)}^{i}$, then

$$
\Delta_{P_{1}}(Q) \nabla_{P_{2}}=\sum\left\langle P_{1}, Q_{(1)}^{i}\right\rangle \cdot Q_{(2)}^{i} \cdot\left\langle Q_{(3)}^{i}, P_{2}\right\rangle
$$

2.3.4. Antipode. The antipode $S: \mathcal{E}_{n} \rightarrow \mathcal{E}_{n}$ is defined by $x_{i j} \mapsto-x_{i j}$, extended to all of $\mathcal{E}_{n}$ as a braided antihomomorphism. In other words, if $\mu: \mathcal{E}_{n} \otimes \mathcal{E}_{n} \rightarrow \mathcal{E}_{n}$ is the multiplication map, then

$$
S(P Q)=S(\mu(P \otimes Q))=\mu(\tau(S(P) \otimes S(Q)))=\mu\left(S(Q) \otimes s_{Q}^{-1}(S(P))\right)=S(Q) \cdot s_{Q}^{-1}(S(P)) .
$$

The antipode preserves $\mathfrak{S}_{n}$-degree.

Example 2.9. The antipode of $x_{12} x_{23} x_{34}$ is

$$
\begin{aligned}
S\left(x_{12} x_{23} x_{34}\right) & =S\left(x_{34}\right) \cdot s_{34}\left(S\left(x_{12} x_{23}\right)\right) \\
& =S\left(x_{34}\right) \cdot s_{34}\left(S\left(x_{23}\right)\right) \cdot s_{34} s_{23}\left(S\left(x_{12}\right)\right) \\
& =\left(-x_{34}\right) \cdot\left(-x_{24}\right) \cdot\left(-x_{14}\right) \\
& =-x_{34} x_{24} x_{14} .
\end{aligned}
$$

It will be helpful for us to introduce a variant of the antipode. Let $\rho: \mathcal{E}_{n} \rightarrow \mathcal{E}_{n}$ be the map that reverses the order of any monomial, and for any homogeneous $P$, let $\bar{S}(P)=$ $(-1)^{\operatorname{deg} P} \rho(S(P))$. For example, $\bar{S}\left(x_{12} x_{23} x_{34}\right)=x_{14} x_{24} x_{34}$. Note that $s_{\bar{S}(P)}=s_{P}^{-1}$.

From this definition, it is easy to check that $\bar{S}\left(x_{i_{1} j_{1}} \cdots x_{i_{\ell} j_{\ell}}\right)=y_{1} \cdots y_{\ell}$, where $y_{k}=$ $s_{i_{\ell} j_{\ell}} \cdots s_{i_{k+1} j_{k+1}}\left(x_{i_{k} j_{k}}\right)$.

The following proposition gives some important properties of $\bar{S}$.

Proposition 2.10. (a) For homogeneous $P, Q \in \mathcal{E}_{n}, \bar{S}(P Q)=s_{Q}^{-1}(\bar{S}(P)) \cdot \bar{S}(Q)$.

(b) The map $\bar{S}$ is an involution.

(c) Let $\bar{\tau}: \mathcal{E}_{n} \otimes \mathcal{E}_{n} \rightarrow \mathcal{E}_{n} \otimes \mathcal{E}_{n}$ be the linear map that switches the two tensor factors (without twisting). Then $\Delta \circ \bar{S}=\bar{\tau} \circ(\bar{S} \otimes \bar{S}) \circ \Delta$.

(d) For any $P \in \mathcal{E}_{n}, \Delta_{a b}(\bar{S}(P))=\bar{S}\left((P) \nabla_{a b}\right)$.

(e) The operators $\bar{S}$ and $\rho$ are adjoint with respect to $\langle\cdot, \cdot\rangle$.

Proof. For (a),

$$
\begin{aligned}
\bar{S}(P Q) & =(-1)^{\operatorname{deg} P Q} \cdot \rho(S(P Q)) \\
& =(-1)^{\operatorname{deg} P+\operatorname{deg} Q} \cdot \rho\left(S(Q) \cdot s_{Q}^{-1}(S(P))\right. \\
& =(-1)^{\operatorname{deg} P} s_{Q}^{-1}\left(\rho(S(P)) \cdot(-1)^{\operatorname{deg} Q} \rho(S(Q))\right. \\
& =s_{Q}^{-1}(\bar{S}(P)) \cdot \bar{S}(Q) .
\end{aligned}
$$

For (b), we induct on degree. It is clear that $\bar{S}^{2}$ is the identity in degree 0 and 1 . Then for higher degrees,

$$
\bar{S}^{2}(P Q)=\bar{S}\left(s_{Q}^{-1}(\bar{S}(P)) \cdot \bar{S}(Q)\right)=s_{\bar{S}(Q)}^{-1} \bar{S}\left(s_{Q}^{-1}(\bar{S}(P))\right) \cdot \bar{S}^{2}(Q)=s_{Q} s_{Q}^{-1}\left(\bar{S}^{2}(P)\right) \cdot Q=P Q
$$


For (c), we again induct on degree. Again the claim is clear in degree 0 or 1 . Suppose $\Delta(P)=\sum P_{(1)}^{i} \otimes P_{(2)}^{i}$ and $\Delta(Q)=\sum Q_{(1)}^{j} \otimes Q_{(2)}^{j}$. Then by induction,

$$
\begin{aligned}
\Delta \circ \bar{S}(P Q) & =\Delta\left(s_{Q}^{-1}(\bar{S}(P)) \cdot \bar{S}(Q)\right) \\
& =s_{Q}^{-1} \Delta(\bar{S}(P)) \cdot \Delta(\bar{S}(Q)) \\
& =s_{Q}^{-1}(\bar{\tau} \circ(\bar{S} \otimes \bar{S}) \circ \Delta(P)) \cdot \bar{\tau} \circ(\bar{S} \otimes \bar{S}) \circ \Delta(Q) \\
& =\sum_{i, j} s_{Q}^{-1}\left(\bar{S}\left(P_{(2)}^{i}\right) \otimes \bar{S}\left(P_{(1)}^{i}\right)\right) \cdot\left(\bar{S}\left(Q_{(2)}^{j}\right) \otimes \bar{S}\left(Q_{(1)}^{j}\right)\right) \\
& =\sum_{i, j} s_{Q}^{-1}\left(\bar{S}\left(P_{(2)}^{i}\right)\right) \bar{S}\left(Q_{(2)}^{j}\right) \otimes s_{\bar{S}\left(Q_{(2)}^{j}\right)}^{-1}\left(s_{Q}^{-1}\left(\bar{S}\left(P_{(1)}^{i}\right)\right)\right) \bar{S}\left(Q_{(1)}^{j}\right) \\
& =\sum_{i, j} s_{Q_{(2)}^{j}}^{-1} \bar{S}\left(s_{Q_{(1)}^{j}}^{-1}\left(P_{(2)}^{i}\right)\right) \bar{S}\left(Q_{(2)}^{j}\right) \otimes s_{Q_{(1)}^{j}}^{-1}\left(\bar{S}\left(P_{(1)}^{i}\right)\right) \bar{S}\left(Q_{(1)}^{j}\right) \\
& =\sum_{i, j} \bar{S}\left(s_{Q_{(1)}^{j}}^{-1}\left(P_{(2)}^{i}\right) Q_{(2)}^{j}\right) \otimes \bar{S}\left(P_{(1)}^{i} Q_{(1)}^{j}\right) \\
& =\sum_{i, j} \bar{\tau} \circ(\bar{S} \otimes \bar{S})\left(P_{(1)}^{i} Q_{(1)}^{j} \otimes s_{Q_{(1)}^{j}}^{-1} P_{(2)}^{i} Q_{(2)}^{j}\right) \\
& =\bar{\tau} \circ(\bar{S} \otimes \bar{S}) \circ \Delta(P Q) .
\end{aligned}
$$

For $(d)$,

$$
\begin{aligned}
\Delta \circ \bar{S}(P) & =\bar{\tau} \circ(\bar{S} \otimes \bar{S}) \circ \Delta(P) \\
& =\sum_{i} \bar{\tau} \circ(\bar{S} \otimes \bar{S})\left(P_{(1)}^{i} \otimes P_{(2)}^{i}\right) \\
& =\sum_{i} \bar{S}\left(P_{(2)}^{i}\right) \otimes \bar{S}\left(P_{(1)}^{i}\right) .
\end{aligned}
$$

Thus

$$
\Delta_{a b}(\bar{S}(P))=\sum_{i}\left\langle x_{a b}, \bar{S}\left(P_{(2)}^{i}\right)\right\rangle \cdot \bar{S}\left(P_{(1)}^{i}\right)=\sum_{i}\left\langle x_{a b}, P_{(2)}^{i}\right\rangle \cdot \bar{S}\left(P_{(1)}^{i}\right)=\bar{S}\left((P) \nabla_{a b}\right) .
$$

For (e), if $P$ and $Q$ are homogeneous of the same degree, then

$$
\langle Q, \bar{S}(P)\rangle=\Delta_{Q}(\bar{S}(P))=\bar{S}\left((P) \Delta_{\rho(Q)}\right)=(P) \Delta_{\rho(Q)}=\langle P, \rho(Q)\rangle .
$$

Remark 2.11. Propostion 2.10(e) easily implies that the antipode $S$ is self-adjoint, but we will not need this result below.

Since we did not use any of the relations of $\mathcal{E}_{n}$ in proving Proposition 2.10, the analogous result holds even in the full tensor algebra with no relations. In particular, for part (c), if $P$ is a monomial of degree $d$, then the $2^{d}$ terms obtained from expanding $\Delta \circ \bar{S}(P)$ are identical to the $2^{d}$ terms obtained from expanding $\bar{\tau} \circ(\bar{S} \otimes \bar{S}) \circ \Delta(P)$ without using any relations between the $x_{i j}\left(\right.$ even $\left.x_{i j}=-x_{j i}\right)$.

In the next section, we will use the properties presented above to prove Theorem 2.4. 


\section{Positivity}

Let us denote by $\mathcal{E}_{n}^{+}$the positive span of monomials in variables $x_{i j}$ with $i<j$. In this section, we will prove that $x_{w / v} \in \mathcal{E}_{n}^{+}$as conjectured by Kirillov [9]. This will immediately imply Theorem 2.4.

First we relate the operators and pairing described in the previous section to the Bruhat order.

Proposition 3.1. (a) Let $w \in \mathfrak{S}_{n}$, and choose any $x_{i j} \in \mathcal{E}_{n}^{+}$. Then $\left(x_{w}\right) \nabla_{i j}=x_{w s_{i j}}$ if $w s_{i j} \lessdot w$ and 0 otherwise.

(b) Let $v, w \in \mathfrak{S}_{n}$. Then $\left(x_{w}\right) \nabla_{v}=x_{v^{\prime}}$ if there exists a reduced factorization $w=v^{\prime} \cdot v^{-1}$ and 0 otherwise.

Proof. Let $w=s_{a_{1}} \cdots s_{a_{\ell}}$. From the definition of coproduct, the degree $(\ell-1,1)$ part of $\Delta\left(x_{w}\right)$ is

$$
\sum_{k=1}^{\ell} x_{a_{1}, a_{1}+1} \cdots \widehat{x}_{a_{k}, a_{k}+1} \cdots x_{a_{\ell}, a_{\ell}+1} \otimes s_{a_{\ell}} \cdots s_{a_{k+1}}\left(x_{a_{k}, a_{k}+1}\right) .
$$

The first tensor factor is nonzero if and only if $s_{a_{1}} \cdots \widehat{s}_{a_{k}} \cdots s_{a_{\ell}}$ is a reduced expression for some $v \in \mathfrak{S}_{n}$ with $v \lessdot w$, and in this case, the second tensor factor must be, up to sign, $x_{v^{-1} w}$.

If $v \lessdot w$, then by the strong exchange condition for Bruhat order, there is a unique $k$ such that $s_{a_{1}} \cdots \widehat{s}_{a_{k}} \cdots s_{a_{\ell}}$ is a reduced expression for $v$. Hence $\left(x_{w}\right) \nabla_{i j}=0$ unless $v=w s_{i j} \lessdot w$, in which case $\left(x_{w}\right) \nabla_{i j}=x_{v} \cdot\left\langle s_{a_{\ell}} \cdots s_{a_{k+1}}\left(x_{a_{k}, a_{k}+1}\right), x_{i j}\right\rangle= \pm x_{v}$. But in fact this must have a positive sign: since $s_{a_{k}} \cdot s_{a_{k+1}} \cdots s_{a_{\ell}}>s_{a_{k+1}} \cdots s_{a_{\ell}}$, it follows that $s_{a_{\ell}} \cdots s_{a_{k+1}}\left(a_{k}\right)<$ $s_{a_{\ell}} \cdots s_{a_{k+1}}\left(a_{k}+1\right)$, so the former must be $i$ and the latter $j$ (since $i<j$ by assumption).

For part (b), choose any reduced expression for $v$ and apply part (a) repeatedly.

By iterating this result, we can prove the following consequence.

Proposition 3.2. Let $w \in \mathfrak{S}_{n}$, and let $P=x_{i_{1} j_{1}} \ldots x_{i_{\ell} j_{\ell}} \in \mathcal{E}_{n}^{+}$be any monomial. Let $v_{k}=s_{i_{k} j_{k}} s_{i_{k+1} j_{k+1}} \cdots s_{i_{\ell} j_{\ell}}$. Then $\left\langle x_{w}, P\right\rangle=1$ if

$$
\text { id } \lessdot v_{\ell} \lessdot v_{\ell-1} \lessdot \cdots \lessdot v_{1}=w^{-1}
$$

is a saturated chain in the Bruhat order of $\mathfrak{S}_{n}$; otherwise $\left\langle x_{w}, P\right\rangle=0$.

In particular, for $v, w \in \mathfrak{S}_{n},\left\langle x_{v}, x_{w}\right\rangle=1$ if $w=v^{-1}$ and 0 otherwise.

Proof. Clearly for $\left\langle x_{w}, P\right\rangle$ to be nonzero, we must have $\ell=\ell(w)=\operatorname{deg}(P)$. We proceed by induction on $\ell$.

Note $\left\langle x_{w}, P\right\rangle=\left\langle\left(x_{w}\right) \nabla_{i_{1} j_{1}}, P^{\prime}\right\rangle$, where $P=x_{i_{1} j_{1}} P^{\prime}$. This is nonzero if and only if, by Proposition 3.1, $w s_{i_{1} j_{1}} \lessdot w$ and, by induction, id $\lessdot v_{\ell} \lessdot v_{\ell-1} \lessdot \cdots \lessdot v_{2}=\left(w s_{i_{1} j_{1}}\right)^{-1}$ is a saturated Bruhat chain, in which case $\left\langle x_{w}, P\right\rangle=1$. But then

$$
v_{2}=s_{i_{1} j_{1}} w^{-1} \lessdot w^{-1}=s_{i_{1} j_{1}} v_{2}=v_{1},
$$

completing the Bruhat chain, as desired.

We will also need the following result about $\bar{S}\left(x_{w}\right)$.

Proposition 3.3. Let $w \in \mathfrak{S}_{n}$. Then $\bar{S}\left(x_{w}\right) \in \mathcal{E}_{n}^{+}$. In fact, the variables appearing in $\bar{S}\left(x_{w}\right)$ are precisely those $x_{i j}$ for which $i<j$ and $w(i)>w(j)$. 
Proof. Let $w=s_{i_{1}} \ldots s_{i_{\ell}}$ be a reduced expression, and let $w_{k}=s_{i_{k}} \cdots s_{i_{\ell}}$. Then by definition, $\bar{S}\left(x_{w}\right)=y_{1} \cdots y_{\ell}$, where $y_{k}=w_{k+1}^{-1}\left(x_{i_{k}, i_{k}+1}\right)$. Since $w_{k+1}^{-1} s_{i_{k}}=w_{k}^{-1}>w_{k+1}^{-1}$, we must have $a=w_{k+1}^{-1}\left(i_{k}\right)<w_{k+1}^{-1}\left(i_{k}+1\right)=b$, so $y_{k}=x_{a b} \in \mathcal{E}_{n}^{+}$. Then $w_{k}(a)=i_{k}+1>i_{k}=w_{k}(b)$. Since multiplying on the left by simple transpositions cannot remove inversions without decreasing length, it follows that $w(a)>w(b)$, as desired.

Proposition 3.3 implies that, in particular, $\bar{S}\left(x_{w_{0}}\right) \in \mathcal{E}_{n}^{+}$. In fact, we can say more about $\bar{S}\left(x_{w_{0}}\right)$. This will be related to the following definition from the theory of Coxeter groups (see [2, 4]).

Definition. A reflection ordering for the transpositions $s_{i j} \in \mathfrak{S}_{n}$ is a total order $\prec$ such that for any $i<j<k, s_{i k}$ lies somewhere between $s_{i j}$ and $s_{j k}$.

There is an equivalent formulation: $t_{1} \prec \cdots \prec t_{N}$ is a reflection ordering if and only if there exists a reduced expression $w_{0}=s_{i_{1}} \cdots s_{i_{N}}$ such that $t_{k}=s_{i_{N}} \cdots s_{i_{k+1}} s_{i_{k}} s_{i_{k+1}} \ldots s_{i_{N}}$. In other words, if $s_{i_{1} j_{1}} \prec \cdots \prec s_{i_{N} j_{N}}$ is a reflection ordering, then $x_{i_{1} j_{1}} \cdots x_{i_{N} j_{N}}=\bar{S}\left(x_{w_{0}}\right)$. We are now ready to prove the following proposition.

Proposition 3.4. In $\mathcal{E}_{n}, x_{w_{0}}=\bar{S}\left(x_{w_{0}}\right)=x_{i_{1} j_{1}} \cdots x_{i_{N} j_{N}} \in \mathcal{E}_{n}^{+}$, where $s_{i_{1} j_{1}} \prec \cdots \prec s_{i_{N} j_{N}}$ is any reflection ordering.

Proof. It suffices to prove the claim for a fixed reflection ordering, say

$$
s_{12} \prec s_{13} \prec s_{23} \prec s_{14} \prec s_{24} \prec s_{34} \prec \cdots \prec s_{1 n} \prec \cdots \prec s_{n-1, n} .
$$

We induct on $n$. Let $w_{0}^{\prime}$ be the longest element of $S_{n-1}$, so $w=w_{0}^{\prime} \cdot s_{n-1} s_{n-2} \cdots s_{1}$ is a reduced factorization. By the inductive hypothesis, $x_{w_{0}^{\prime}}=x_{12} x_{13} x_{23} \cdots x_{n-2, n-1} \in \mathcal{E}_{n-1}$. Since $w_{0}^{\prime}$ has maximum length in $S_{n-1}, x_{w_{0}^{\prime}} x_{i, i+1}=0$ for $i=1, \ldots, n-2$. Then

$$
\begin{aligned}
x_{w_{0}^{\prime}} \cdot x_{1 n} x_{2 n} x_{3 n} \cdots x_{n-1, n} & =x_{w_{0}^{\prime}} \cdot\left(-x_{12} x_{1 n}+x_{2 n} x_{12}\right) \cdot x_{3 n} \cdots x_{n-1, n} \\
& =x_{w_{0}^{\prime}} \cdot x_{2 n} x_{12} \cdot x_{3 n} \cdots x_{n-1, n} \\
& =x_{w_{0}^{\prime}} \cdot x_{2 n} x_{3 n} \cdots x_{n-1, n} \cdot x_{12} \\
& =x_{w_{0}^{\prime}} \cdot\left(-x_{23} x_{2 n}+x_{3 n} x_{23}\right) \cdots x_{n-1, n} \cdot x_{12} \\
& =x_{w_{0}^{\prime}} \cdot x_{3 n} \cdots x_{n-1, n} \cdot x_{23} x_{12} \\
& =\cdots \\
& =x_{w_{0}^{\prime}} \cdot x_{n-1, n} x_{n-2, n-1} \cdots x_{23} x_{12} \\
& =x_{w_{0}} .
\end{aligned}
$$

We are now ready to prove our main theorem.

Theorem 3.5. For any $v, w \in \mathfrak{S}_{n}, x_{w / v} \in \mathcal{E}_{n}^{+}$.

Proof. Since $\ell\left(w_{0}\right)=\ell(w)+\ell\left(\left(w_{0} w\right)^{-1}\right)$, by Proposition $3.1(\mathrm{~b}),\left(x_{w_{0}}\right) \nabla_{w_{0} w}=x_{w}$. Then by Propositions 2.7 and [3.2, $\Delta_{v^{-1}}\left(x_{w}\right)=\sum_{v^{\prime}}\left\langle x_{v^{-1}}, x_{v^{\prime}}\right\rangle \cdot x_{w / v^{\prime}}=x_{w / v}$. Thus

$$
x_{w / v}=\Delta_{v^{-1}}\left(x_{w_{0}}\right) \nabla_{w_{0} w} .
$$

Now by Propositions 3.4, 2.10(d), and 3.1](b),

$$
\Delta_{v^{-1}}\left(x_{w_{0}}\right)=\Delta_{v^{-1}}\left(\bar{S}\left(x_{w_{0}}\right)\right)=\bar{S}\left(\left(x_{w_{0}}\right) \nabla_{v}\right)=\bar{S}\left(x_{w_{0} v}\right) .
$$


Write $\Delta\left(\bar{S}\left(x_{w_{0} v}\right)\right)=\sum X_{(1)}^{i} \otimes X_{(2)}^{i}$. Then since $\bar{S}\left(x_{w_{0} v}\right) \in \mathcal{E}_{n}^{+}$by Proposition 3.3, we also have $X_{(1)}^{i} \in \mathcal{E}_{n}^{+}$. Moreover, by Propositions 2.10(c) and 2.7,

$$
\sum X_{(2)}^{i} \otimes X_{(1)}^{i}=\bar{\tau} \circ \Delta \circ \bar{S}\left(x_{w_{0} v}\right)=(\bar{S} \otimes \bar{S}) \circ \Delta\left(x_{w_{0} v}\right)=\sum_{u} \bar{S}\left(x_{u}\right) \otimes \bar{S}\left(x_{w_{0} v / u}\right) .
$$

Hence if $X_{(2)}^{i}$ is nonzero, then it equals $\bar{S}\left(x_{u}\right)$ for some $u \in \mathfrak{S}_{n}$ and therefore lies in $\mathcal{E}_{n}^{+}$. Then

$$
x_{w / v}=\left(\bar{S}\left(x_{w_{0} v}\right)\right) \nabla_{w_{0} w}=\sum X_{(1)}^{i} \cdot\left\langle X_{(2)}^{i}, x_{w_{0} w}\right\rangle .
$$

Since $\left\langle X_{(2)}^{i}, x_{w_{0} w}\right\rangle$ is either 0 or 1 by Proposition $3.2, x_{w / v}$ lies in $\mathcal{E}_{n}^{+}$, as desired.

Tracing through the proof of Theorem 3.5, we can write down an explicit expression for $x_{w / v}$.

Corollary 3.6. Let $v, w \in \mathfrak{S}_{n}$. Choose any reduced expression $w_{0} v=s_{i_{1}} \cdots s_{i_{\ell}}$, and let $w_{k}=s_{i_{k}} \cdots s_{i_{\ell}}$. Define $y_{k}=w_{k+1}^{-1}\left(x_{i_{k}, i_{k}+1}\right) \in \mathcal{E}_{n}^{+}$. Then

$$
x_{w / v}=\sum_{J} \prod_{k \notin J} y_{k}
$$

where $J \subseteq\{1, \ldots, \ell\}$ ranges over all subsets such that $\prod_{k \in J} s_{i_{k}}$ is a reduced expression for $w_{0} w$.

Proof. As in Theorem 3.5, $x_{w / v}=\left(\bar{S}\left(x_{w_{0} v}\right)\right) \nabla_{w_{0} w}=\left(y_{1} \cdots y_{\ell}\right) \nabla_{w_{0} w}$. By the proof of Theorem 3.5 ,

$$
\Delta\left(y_{1} \cdots y_{\ell}\right)=\sum_{J \subseteq\{1, \ldots, \ell\}}\left(\left(\prod_{k \notin J} y_{k}\right) \otimes \bar{S}\left(\prod_{k \in J} x_{i_{k}, i_{k}+1}\right)\right) .
$$

By Proposition 2.10(e) $\left\langle\bar{S}\left(x_{u}\right), x_{w_{0} w}\right\rangle=\left\langle x_{u}, x_{w^{-1} w_{0}}\right\rangle$, which by Proposition 3.2 equals 1 if $u=w_{0} w$ and 0 otherwise. The result follows.

Example 3.7. Let $w=s_{2} s_{1} s_{3} s_{2} \in \mathfrak{S}_{4}$ and $v=s_{2}$. One reduced expression for $w_{0} v$ is $s_{3} s_{2} s_{1} s_{2} s_{3}$. Then $\bar{S}\left(x_{w_{0} v}\right)=x_{13} x_{12} x_{14} x_{24} x_{34}$. Since $w_{0} w=s_{1} s_{3}=s_{3} s_{1}$, there are two reduced subexpressions for $w_{0} w$ in $w_{0} v$, namely for $J=\{1,3\}$ and $J=\{3,5\}$. Removing the corresponding variables from $\bar{S}\left(x_{w_{0} v}\right)$ gives

$$
x_{w / v}=x_{12} x_{24} x_{34}+x_{13} x_{12} x_{24} .
$$

Compare this calculation to Example 2.3.

Each term in the expansion of $x_{w / v}$ corresponds to a reduced subword for $w_{0} w$ lying inside a reduced expression for $w_{0} v$. For more information about these subwords, see [10].

Interestingly, Corollary 3.6 implies that $x_{w / v}$ has an expression in which no variable is repeated in any monomial.

One can also use Theorem 3.5 to give a positive recurrence for $x_{w / v}$.

Corollary 3.8. Let $v, w \in \mathfrak{S}_{n}$, and suppose $a=v^{-1}(i)<v^{-1}(i+1)=b$. Let $v^{\prime}=s_{i} v$ and $w^{\prime}=s_{i} w$. If $w \lessdot w^{\prime}$, then $x_{w / v}=x_{a b} x_{w / v^{\prime}}+x_{w^{\prime} / v^{\prime}}$; otherwise $x_{w / v}=x_{a b} x_{w / v^{\prime}}$.

Proof. If $v^{-1}(i)<v^{-1}(i+1)$, then $\left(w_{0} v\right)^{-1}(n+1-i)<\left(w_{0} v^{-1}\right)(n-i)$. Hence there exists a reduced expression for $w_{0} v$ that starts with $s_{n-i}$. Let us fix such a reduced expression and apply Corollary 3.6. In this case $y_{1}=x_{a b}$, and $y_{2} \cdots y_{\ell}=\bar{S}\left(x_{s_{n-i} w_{0} v}\right)=\bar{S}\left(x_{w_{0} v^{\prime}}\right)$. 
Suppose $J \subseteq\{1, \ldots, \ell\}$ gives a reduced subexpression for $w_{0} w$. If $1 \notin J$, then $J$ gives a reduced subexpression for $w_{0} w$ in a reduced expression for $w_{0} v^{\prime}$. This then contributes $x_{a b} x_{w / v^{\prime}}$ to $x_{w / v}$. If $1 \in J$, then we must have $w_{0} w^{\prime}=s_{n-i} w_{0} w \lessdot w_{0} w$, or equivalently $w \lessdot w^{\prime}$, and $J \backslash\{1\}$ must give a reduced subexpression for $w_{0} w^{\prime}$ in a reduced expression for $w_{0} v^{\prime}$. Hence this contributes $x_{w^{\prime} / v^{\prime}}$ to $x_{w / v}$ (provided $\left.w \lessdot w^{\prime}\right)$. The result follows.

Corollary 3.8 then gives an explicit positive recurrence that implies $x_{w / v} \in \mathcal{E}_{n}^{+}$(noting that if $\ell(v)=\ell(w)$, then $x_{w / v}=1$ if $v=w$ and 0 otherwise).

\section{REFERENCES}

[1] Nicolás Andruskiewitsch and Hans-Jürgen Schneider. Pointed Hopf algebras. In New directions in Hopf algebras, volume 43 of Math. Sci. Res. Inst. Publ., pages 1-68. Cambridge Univ. Press, Cambridge, 2002.

[2] Anders Björner and Francesco Brenti. Combinatorics of Coxeter groups, volume 231 of Graduate Texts in Mathematics. Springer, New York, 2005.

[3] Jonah Blasiak, Ricky Ini Liu, and Karola Mészáros. Subalgebras of the Fomin-Kirillov algebra. Preprint, 2012. arxiv:1310.4112.

[4] Francesco Brenti, Sergey Fomin, and Alexander Postnikov. Mixed Bruhat operators and Yang-Baxter equations for Weyl groups. Internat. Math. Res. Notices, (8):419-441, 1999.

[5] Sergey Fomin and Anatol N. Kirillov. Quadratic algebras, Dunkl elements, and Schubert calculus. In Advances in geometry, volume 172 of Progr. Math., pages 147-182. Birkhäuser Boston, Boston, MA, 1999.

[6] Sergey Fomin and Claudio Procesi. Fibered quadratic Hopf algebras related to Schubert calculus. $J$. Algebra, 230(1):174-183, 2000.

[7] Sergey Fomin and Richard P. Stanley. Schubert polynomials and the nil-Coxeter algebra. Adv. Math., 103(2):196-207, 1994.

[8] James E. Humphreys. Reflection groups and Coxeter groups, volume 29 of Cambridge Studies in Advanced Mathematics. Cambridge University Press, Cambridge, 1990.

[9] Anatol N. Kirillov. Skew divided difference operators and Schubert polynomials. SIGMA Symmetry Integrability Geom. Methods Appl., 3:Paper 072, 14, 2007.

[10] Allen Knutson and Ezra Miller. Subword complexes in Coxeter groups. Adv. Math., 184(1):161-176, 2004.

[11] I. G. Macdonald. Schubert polynomials. In Surveys in combinatorics, 1991 (Guildford, 1991), volume 166 of London Math. Soc. Lecture Note Ser., pages 73-99. Cambridge Univ. Press, Cambridge, 1991.

[12] Alexander Milinski and Hans-Jürgen Schneider. Pointed indecomposable Hopf algebras over Coxeter groups. In New trends in Hopf algebra theory (La Falda, 1999), volume 267 of Contemp. Math., pages 215-236. Amer. Math. Soc., Providence, RI, 2000.

Department of Mathematics, North Carolina State University, Raleigh, NC

E-mail address: riliu@ncsu.edu 